\title{
Thalidomide for Treatment of Immune Reconstitution Inflammatory Syndrome in a HIV Patient with Cutaneous Mycobacterium avium Complex Infection
}

\author{
Yirui Xie ${ }^{1-3}$, Jia Sun ${ }^{1-3}$, Junwei Su ${ }^{1-3}$, Lijun $\mathrm{Xu}^{1-3}$, Ying Huang ${ }^{1-3}$ and Biao Zhu ${ }^{1-3 *}$ \\ ${ }^{1}$ State Key Laboratory for Diagnosis and Treatment of Infectious Diseases, China \\ ${ }^{2}$ The First Affiliated Hospital, School of Medicine, Zhejiang University, China \\ ${ }^{3}$ Collaborative Innovation Center for Diagnosis and Treatment of Infectious Diseases, Hangzhou, China
}

\begin{abstract}
Immune reconstitution inflammatory syndrome (IRIS) is a severe clinical complication emerging during the initial months of antiretroviral therapy (ART) in HIV infected patients. Mycobacterium avium complex (MAC) associated IRIS typically develops in severely immunosuppressed individuals, who have an excellent response to ART. Cutaneous MAC infection is uncommon. The skin lesions almost invariably responded well to specific anti-mycobacterial treatment for MAC infection in IRIS patients. Here, we report a case whose cutaneous MAC lesions continue exacerbation despite with anti-mycobacterial treatment. But rapid clinical remission occurred after thalidomide adding to the antituberculosis treatment. Furthermore, immunomodulatory effect of thalidomide on cytokine levels such as decreasing of TNF- $\alpha$, increasing of IL-4 and IL-6 were observed in the patient. Thalidomide may be an effective treatment for cutaneous MAC infection during IRIS. This is the first report of thalidomide treatment for cutaneous MAC infection during IRIS, and needs further mechanism and clinical trial investigation in this regard.
\end{abstract}

\section{Keywords:}

\section{Introduction}

Immune reconstitution inflammatory syndrome (IRIS) is a severe clinical complication emerging during the initial months of antiretroviral therapy (ART) in HIV infected patients. The hallmark of IRIS is paradoxical worsening of an existing infection/disease process or appearance of a new infection/disease process after initiation of ART [1]. The reported incidence of non-tuberculous mycobacterial (NTM) associated IRIS is $3.5 \%$ among patients with baseline $\mathrm{CD} 4$ cell count $<100$ cells/ $\mu \mathrm{l}$ [2]. Mycobacterium avium complex associated IRIS (MAC-IRIS) typically develops in severely immunosuppressed individuals, who have an excellent response to ART [3]. In contrast to disseminated MAC disease of advanced AIDS, MAC-IRIS usually presents as localized disease [2,3]. The most common manifestation of MACIRIS is fever with suppurative painful lymphadenitis (69\%), followed by pulmonary disease (19\%) [3]. Cutaneous MAC infection is uncommon, and it is not easily differentiated from other chronic skin lesions [4]. The skin lesions almost invariably responded well to specific anti-mycobacterial treatment for MAC infection in IRIS patients. Here, we report a case whose cutaneous MAC lesions continue exacerbation despite with anti-mycobacterial treatment. But rapid clinical remission occurred after thalidomide adding to the anti-tuberculosis treatment.

\section{Case Report}

A 61-year-old Chinese man was admitted to the Infectious Diseases Unit in August 2013. He presented with symptoms of fever and anorexia for 40 days. The man was diagnosed with HIV infection (CD4 cell count was 18 cells/ $\mu$ l and HIV-1 plasma viral load was 40,346 copies/ $\mathrm{ml}$ ). Pulmonary CT scan showed atelectasis and variable-sized nodular lesions in the right upper lobe of lung. Right hilar and mediastinal lymphadenopathy were also observed (Figure 1a). Acid-fast bacilli were found in the sputum suggested the diagnosis of tuberculosis. So antimycobacterial therapy (isoniazid $[\mathrm{H}]$, rifampicin [R], pyrazinamide $[\mathrm{Z}]$, and ethambutol [E]) was introduced 2 days after admission. His fever resolved and appetite improved one week later.
Ten weeks after starting antitubercular treatment, he initiated combination antiretroviral therapy- Zidovudine (AZT, $300 \mathrm{mg}$, twice daily), lamivudine (3TC, $300 \mathrm{mg}$, daily), and efavirenz (EFV, 600 mg, at night). However, he manifested with high fever, cough, thick white sputum, and shortness of breath one week later. His CD4 count had increased to 114 cells/ $\mu$ l and HIV viral load was 4,242 copies/ ml. pulmonary lesions increased on CT scan (Figure 1b). Tumoral, autoimmune, infection and iatrogenic causes of fever were excluded. This strongly suggested IRIS, so thalidomide (100 mg, once daily) was introduced at November 20, 2013. His fever disappeared and appetite improved one week later. The symptom of cough, sputum, shortness of breath and fever disappeared two weeks later. Pulmonary CT scan showed atelectasis increased but variable-sized nodular lesions absorbed significantly at December 13, 2013 (Figure 1c). However, he discontinued thalidomide and aspirin two months later at January, 2014.

One month later after stop using thalidomide, a walnut-sized lump appeared below the patient's thyroid cartilage and on the right submandibular respectively. Ultrasound examinations showed the mass below thyroid cartilage was $3.7 \times 3.2 \mathrm{~cm}$ (Figure 2a); the mass on the right submandibular was $2.9 \times 1.7 \mathrm{~cm}$ (Figure $2 \mathrm{~b}$ ). The lump below the thyroid cartilages ulcerated at February, 2014. Caseous necrotic

*Corresponding author: Biao Zhu, Department of Infectious Diseases, The First Affiliated Hospital of Medical School, Zhejiang University, 79 QingChun Road, Hangzhou, China, 310006, Tel: 86-571-87236437; Fax: 86-571-87236755; E-mail: zhubiao1207@zju.edu.cn

Received November 03, 2015; Accepted December 09, 2015; Published December 14, 2015

Citation: Xie Y, Sun J, Su J, Xu L, Huang Y, et al. (2015) Thalidomide for Treatment of Immune Reconstitution Inflammatory Syndrome in a HIV Patient with Cutaneous Mycobacterium avium Complex Infection. J AIDS Clin Res 6: 530 doi:10.4172/2155-6113.1000530

Copyright: (c) $2015 \mathrm{Xie} \mathrm{Y}$, et al. This is an open-access article distributed unde the terms of the Creative Commons Attribution License, which permits unrestricted use, distribution, and reproduction in any medium, provided the original author and source are credited. 


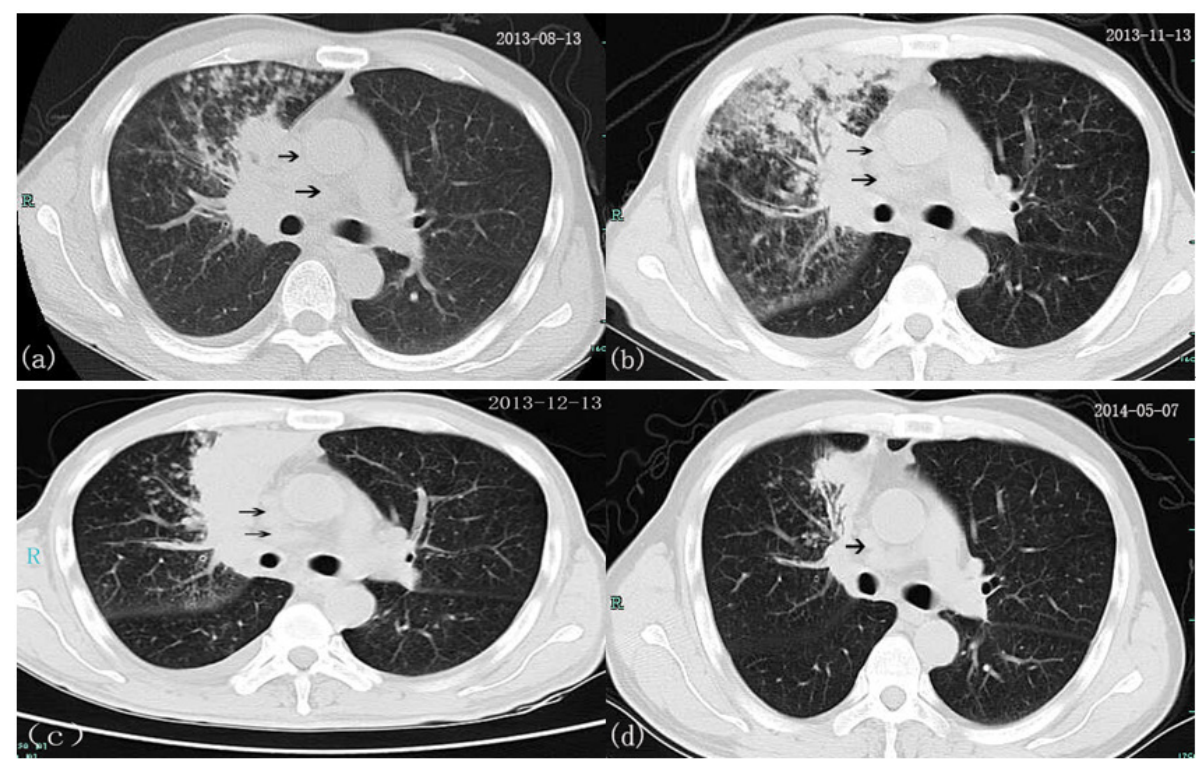

Figure 1: Pulmonary CT.

(a) Pulmonary lesions and lymphadenopathy (arrow) before treatment in August 2013.

(b) Increase of pulmonary lesions after ART in November 2013.

(c) Decrease of pulmonary lesions and lymphadenopathy (arrow) after using thalidomide in December 2013.

(d) Major decrease of pulmonary lesions and lymphadenopathy (arrow) after reusing thalidomide in May 2014.
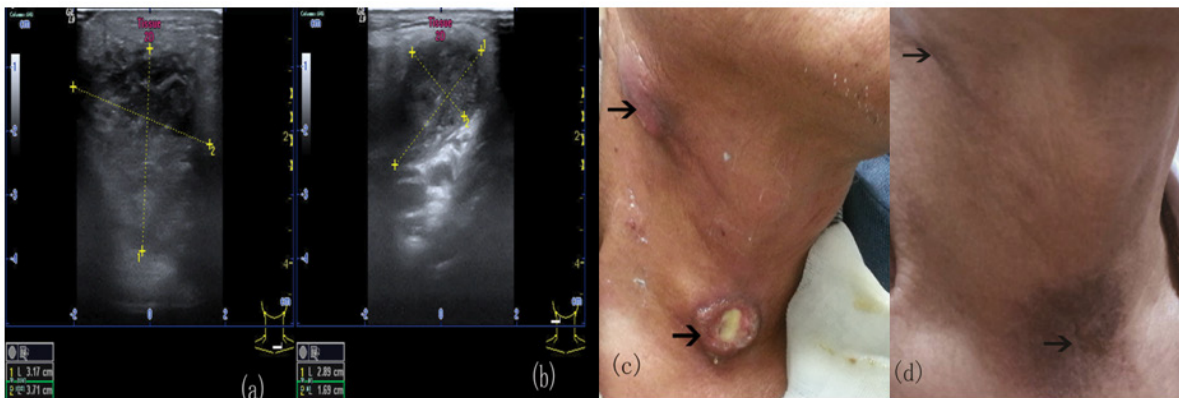

Figure 2: Ultrasound examinations and skin lesions of MAC infection.

(a) The ultrasound examinations of the mass below thyroid cartilage $\left(3.7^{\star} 3.2 \mathrm{~cm}\right)$.

(b) The ultrasound examinations of the mass on the right submandibular $\left(2.9^{*} 1.7 \mathrm{~cm}\right)$.

(c) MAC lesions before thalidomide reusing

(d) Resolution of MAC skin lesions after two months of thalidomide reusing.

tissue was visible on the ulceration (Figure 2c). AZT was changed to TDF (300 mg, daily) because of severe anemia at February 25, 2014. The mycobacterial culture of the tissue show the bacteria was NTM , then CapitalBio ${ }^{\text {Tm }}$ DNA microarray (CapitalBio Corp., Beijing, China) was used to confirmed the diagnosis of MAC infection at March 11, 2014. The patient had history of sever gastrointestinal side effect when using Azithromycin, So the antimycobacterial therapy (Rifabutin 450 mg per day, isoniazid $(\mathrm{H})$ and ethambutol $(\mathrm{E}))$ was initiated at March 11,2014 . However the ulceration continued increasing. Unfortunately, the patient disagreed to do pulmonary CT again. After obtaining informed consent from the patient, thalidomide ( $100 \mathrm{mg}$, once daily) were introduced again at April 1, 2014. The lump below the thyroid cartilage partially healed, and the lump on the right submandibular reduced half month later. Lymphadenopathy and atelectasis reduced obviously in pulmonary CT one month later (Figure 1d). Interestingly, the ulceration disappeared two months later after thalidomide reusing (Figure 2d).
We retrospectively quantified the cytokine concentrations of TNF- $\alpha$, IL-2, IL-4, IL-6, IL-10, IL-12, IL-17a, IFN- $\gamma$ and MCP-1 in his serum using ELISA (eBioscience, San Diego, CA, USA) during and after introduction of thalidomide. The local ethical committee approved the protocol and informed consent was obtained from the patient. We found that thalidomide did not affect IL-2, IL-10, IL-12, IL-17a, and MCP-1(data not shown) levels significantly. However, the plasma level of IL-4, IL- 6 was significantly increased after using thalidomide, but decreased when stop using thalidomide (Figure 3). IFN- $\gamma$ increased significantly after reusing thalidomide. The plasma level of TNF- $\alpha$ was significantly decreased after using thalidomide, but increased when stop using thalidomide (Table 1).

\section{Discussion}

Mycobacterium avium complex (MAC) classified as Runyon group III, non-chromogenic mycobacteria. MAC has been isolated from various environmental sources, but it was rarely identified as a cause 


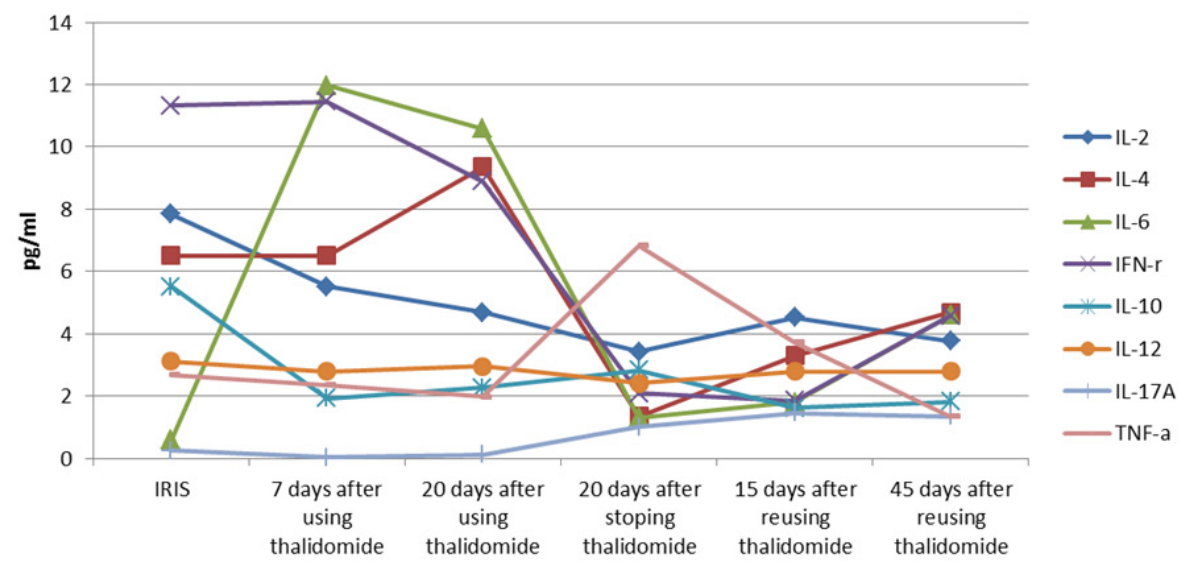

Figure 3: Dynamic of serum cytokines during and after using thalidomide.

\begin{tabular}{|l|c|c|c|c|c|c|c|c|}
\hline & IL-2 & IL-4 & IL-6 & IFN-r & IL-10 & IL-12 & IL-17A & TNF-a \\
\hline Normal ranges $(\mathrm{pg} / \mathrm{ml})$ & $0.1-4.1$ & $0.1-3.2$ & $0.1-2.9$ & $0.1-5.0$ & $0.1-18$ & $0.1-3.1$ & $0.1-2.9$ & $0.1-23$ \\
\hline IRIS & 7.85 & 6.5 & 0.59 & 11.32 & 5.51 & 3.11 & 0.24 & 2.68 \\
\hline 7 days after using thalidomide & 5.51 & 6.5 & 11.97 & 11.45 & 1.93 & 2.79 & 0.03 & 2.35 \\
\hline 20 days after using thalidomide & 4.68 & 9.35 & 10.59 & 8.89 & 2.26 & 2.95 & 0.11 & 1.98 \\
\hline 20 days after stopping thalidomide & 3.42 & 1.34 & 1.31 & 2.09 & 2.83 & 2.41 & 1.01 & 6.84 \\
\hline 15 days after reusing thalidomide & 4.53 & 3.3 & 1.79 & 1.85 & 1.61 & 2.79 & 1.46 & 3.72 \\
\hline 45 days after reusing thalidomide & 3.77 & 4.69 & 4.59 & 4.58 & 1.82 & 2.79 & 1.35 & 1.35 \\
\hline
\end{tabular}

Table 1: Dynamic changes of cytokines in the course of disease.

of disease [5]. The most common presentation of MAC infection is suppurative painful lymphadenitis and pulmonary disease. MAC may also involve other areas, such as the joints, spine and soft tissue, and less commonly, the skin [2]. Cutaneous MAC lesions can include nodules, soft tissue swelling, ulcerations, abscesses, folliculitis, panniculitis, and erythematous lesions with or without granulomas [6]. Just as the patient, cutaneous MAC infection often develop from a single nodule or multiple nodules progressing over time into ulcerative lesions with or without sero-sanguineous exudates.

The skin lesions almost invariably responded well to specific antimycobacterial treatment for MAC infection in IRIS patients [3]. In this case, the cutaneous MAC lesions continue exacerbation despite with anti-mycobacterial treatment. Pathogenic markers related with IRIS include elevations of a broad range of pro-inflammatory cytokines, specific T cell numbers and presence of innate immune cells at the site of disease $[7,8]$. So in patients with severe IRIS, it may be necessary to consider immunomodulatory or anti-inflammatory treatment in addition to the optimizing therapy for underlying pathogen, in order to reduce inflammation, tissue damage and alleviate symptoms. Although corticosteroids have been used in treatment of IRIS, they were associated with the arising risks of further immune-suppression and infections or reactivation of latent infections in already immunesuppressed patients $[9,10]$.

Thalidomide is an anti-inflammatory, immunomodulatory and anti-angiogenic agent [11]. It has already been effectively used in Kaposi sarcoma, aphthous ulcers, gastrointestinal lesions and cryptococcal meningitis in HIV patients. Thalidomide has been also used in isolated cases of IRIS such as tuberculosis IRIS [12,13] and cryptococcus lymphadenitis IRIS [14]. Thalidomide may be a salvage treatment in patients with corticosteroid-dependent or refractory IRIS [15]. Furthermore, use of thalidomide for MAC infection has been reported in patients with AIDS [16] as well as in a HIV-negative patient [17]. However, its effect on the paradoxical reaction in cutaneous MAC infection is not yet clear. In the case, clinical and pulmonary CT responses were rapid under thalidomide treatment, arguing for a direct and strong anti-inflammatory effect of thalidomide. Thalidomide is reported to lack direct activity against MAC [18]. However, it reports that thalidomide can inhibit TNF- $\alpha$ and enhance antigen-specific immunity [11]. Here, immunomodulatory effect of thalidomide on cytokine levels such as decreasing of TNF- $\alpha$ and increasing of IL-4, IL-6 were observed in the patient with MAC-IRIS. The increase of IL-6 has been associated with higher risk for cardiovascular disease in HIV infected patient [19]. In tuberculosis patients with or without concomitant HIV infection, thalidomide treatment was found to both decrease plasma TNF- $\alpha$ protein levels as well as monocyte TNF- $\alpha$ mRNA levels $[20,21]$. However, no obvious immunomodulatory effect of thalidomide on cytokine levels were observed in HIV-infected patients with corticosteroid-dependent disseminated tuberculosis or cryptococcal meningitis associated paradoxical IRIS [15]. Further studies are needed for more detailed understanding of the immunity effect of thalidomide in IRIS. The drug was well tolerated at low doses, with only mild dizziness in the patient. The same situation was observed in HIV-infected patients with corticosteroid-dependent disseminated tuberculosis associated paradoxical IRIS [15]. This case supports further exploration of thalidomide as adjunct therapy for HIV-infected IRIS patients with cutaneous MAC infection.

In conclusion, thalidomide may be an effective treatment for cutaneous MAC infection during IRIS, and it needs further mechanism and clinical trial investigation in this regard.

\section{Acknowledgement}

This work was funded by National Special Research Program for Important Infectious Diseases (No.2013ZX10001004-002-003, 2014ZX10001002-002-002). 
Citation: Xie Y, Sun J, Su J, Xu L, Huang Y, et al. (2015) Thalidomide for Treatment of Immune Reconstitution Inflammatory Syndrome in a HIV Patient with Cutaneous Mycobacterium avium Complex Infection. J AIDS Clin Res 6: 530. doi:10.4172/2155-6113.1000530

Page 4 of 4

\section{References}

1. Muller M, Wandel S, Colebunders R, Attia S, Furrer H, et al. (2010) Immune reconstitution inflammatory syndrome in patients starting antiretroviral therapy for HIV infection: a systematic review and meta-analysis. Lancet Infect Dis 10: 251-261.

2. Phillips P, Bonner S, Gataric N, Bai T, Wilcox P, et al. (2005) Nontuberculous mycobacterial immune reconstitution syndrome in HIV-infected patients: spectrum of disease and long-term follow-up. Clin Infect Dis 41: 1483-1497.

3. Lawn SD, Bekker LG, Miller RF (2005) Immune reconstitution disease associated with mycobacterial infections in HIV-infected individuals receiving antiretrovirals. Lancet Infect Dis. 5: 361-373.

4. Tandon R, Kim KS, Serrao R (2007) Disseminated Mycobacterium aviumintracellulare infection in a person with AIDS with cutaneous and CNS lesions. AIDS Read 17: 555-560.

5. Ohse H, Saito T, Kadono K, Hirano K, Watanabe S, et al. (1997) A case of acquired immunodeficiency syndrome with disseminated Mycobacterium avium complex infection in which $M$. avium was isolated from bone marrow. Kekkaku 72: 73-77.

6. Friedman BF, Edwards D, Kirkpatrick CH (1988) Mycobacterium aviumintracellulare: cutaneous presentations of disseminated disease. Am J Med 85: 257-263.

7. Seddiki N, Sasson SC, Santner-Nanan B, Munier M, van Bockel D, et al. (2009) Proliferation of weakly suppressive regulatory CD4+ T cells is associated with over-active CD4+ T-cell responses in HIV-positive patients with mycobacterial immune restoration disease. Eur J Immunol 39: 391-403.

8. Tadokera R, Meintjes G, Skolimowska KH, Wilkinson KA, Matthews $\mathrm{K}$, et al. (2011) Hypercytokinaemia accompanies HIV-tuberculosis immune reconstitution inflammatory syndrome. Eur Respir J 37: 1248-1259.

9. Venkataramana A, Pardo CA, McArthur JC, Kerr DA, Irani DN, et al. (2006) Immune reconstitution inflammatory syndrome in the CNS of HIV-infected patients. Neurology 67: 383-388.

10. Dalessandro L, Bottaro E (2002) Reactivation of CMV retinitis after treatment with subtenon corticosteroids for immune recovery uveitis in a patient with AIDS. Scandinavian journal of infectious diseases 34: 780-782.
11. Franks ME, Macpherson GR, Figg WD (2004) Thalidomide. Lancet 363: 1802 1811.

12. Fernandes GC, Vieira MA, Lourenço MC, Gadelha AJ, Coura LC, et al. (2002) Inflammatory paradoxical reaction occurring in tuberculosis patients treated with HAART and rifampicin. Rev Inst Med Trop Sao Paulo 44: 113-114.

13. Fourcade C, Mauboussin JM, Lechiche C, Lavigne JP, Sotto A (2014) Thalidomide in the treatment of immune reconstitution inflammatory syndrome in HIV patients with neurological tuberculosis. AIDS patient care and STDs 28 : 567-569.

14. Lortholary O, Fontanet A, Memain N, Martin A, Sitbon K, et al. (2005) Incidence and risk factors of immune reconstitution inflammatory syndrome complicating HIV-associated cryptococcosis in France. AIDS 19: 1043-1049.

15. Brunel AS, Reynes J, Tuaillon E, Rubbo PA, Lortholary O, et al. (2012) Thalidomide for steroid-dependent immune reconstitution inflammatory syndromes during AIDS. AIDS 26: 2110-2112.

16. Bouza E, Muñoz P, Diaz MD, Vicente T (1992) Thalidomide in patients with acquired immunodeficiency syndrome. Arch Intern Med 152: 1089-1090.

17. Gori A, Franzetti F, Marchetti G, Rossi C, Fusi ML, et al. (1998) Clinical and immunological improvement in a patient who received thalidomide treatment for refractory Mycobacterium avium complex infection. Clin Infect Dis 26: 184185.

18. Vicente T, Ortega A, Muñoz P, Diaz MD, Bouza E (1993) In vitro activity of thalidomide against Mycobacterium avium complex. Arch Intern Med 153: 534.

19. Kuller LH, Tracy R, Belloso W, De Wit S, Drummond F, et al. (2008) Inflammatory and coagulation biomarkers and mortality in patients with HIV infection. PLoS Med 5: e203.

20. Tramontana JM, Utaipat U, Molloy A, Akarasewi P, Burroughs M, et al. (1995) Thalidomide treatment reduces tumor necrosis factor alpha production and enhances weight gain in patients with pulmonary tuberculosis. Mol Med 1: 384 397.

21. Klausner JD, Makonkawkeyoon S, Akarasewi P, Nakata K, Kasinrerk W, et al. (1996) The effect of thalidomide on the pathogenesis of human immunodeficiency virus type 1 and $M$. tuberculosis infection. J Acquir Immune Defic Syndr Hum Retrovirol 11: 247-257. 